\title{
Evaluation of Wheat Advanced Lines for Agronomic Traits under Vary Sowing Dates
}

\author{
Arshad Ali ${ }^{1}$, Zia Ullah ${ }^{2 *}$, Nawab Ali $^{2}$ and Muhammad Mehran Anjum ${ }^{2}$ \\ ${ }^{1}$ Department of Agronomy, University of Agricuture Peshawer, Pakistan \\ ${ }^{2}$ Department of Agronomy, University of Agriculture Peshawar, Pakistan
}

Submission: March 10, 2017; Published: May 30, 2017

"Corresponding author: Ziaullah, Department of Agronomy, University of Agriculture Peshawar, Pakistan, Email: ziaullah@aup.edu.pk

\begin{abstract}
Development of new lines in wheat and its evaluation under different planting dates is pre requisites for enhancing productivity on sustainable basis. An experiment was conducted at Cereal Crops Research Institute, Pirsabaq, Now shera Pakistan during 2013-14. The study was carried out to evaluate six wheat advanced lines (PR-103, PR-105, PR-106, PR-107, PR-108 and PR-109) on different planting dates (Oct. 25 , Nov. $5^{\text {th }}$, Nov. $15^{\text {th }}$, Nov. $25^{\text {th }}$, Dec. $5^{\text {th }}$ and Dec. $15^{\text {th }}$ ) to identify the most suitable planting time and judge their performance under late sowing in the central agro-ecological zone of Khyber Pukhtunkhwa (KP), Pakistan. The experiment was laid out in randomized complete block design with split-plot arrangement replicated thrice. Sowing dates effect was studied in main-plots and wheat advance lines in sub-plots. Data were recorded on days to heading, days to maturity, canopy temperature, chlorophyll content, plant height, spike length, spike lets spike ${ }^{-1}$, grains spike ${ }^{-1}$, lodging score (\%), 1000-kernel weight, grain yield, biological yield and harvest index. Highly significant differences were found among planting dates. Days to heading, days to maturity, canopy temperature, chlorophyll content, plant height and spike length of various wheat advance lines were decreased to $17.6,19,11.1,11.515$ and $5 \%$ respectively when the sowing time was delayed from Oct. $25^{\text {th }}$ to Dec. $15^{\text {th }}$. Spikelets spike ${ }^{-1}$, grains spike $^{-1}, 1000$-grain weight, biomass yield, grain yield and harvest index of various wheat advance lines were also decreased to 7.5, 16, 26.7, 47.7, 35.2 and $19.2 \%$ respectively when sowing was delayed from Oct. $25^{\text {th }}$ to Dec. $15^{\text {th }}$, while lodging score\% were maximum on Nov. $5^{\text {th }}$ and zero on Dec. $5^{\text {th }}$. Grain yield losses of 3.6, 9.9, 12.4, 19.4 and $35.2 \%$ were recorded on respective sowing dates and the percent yield losses were increased as sowing delayed from Oct. $25^{\text {th }}$ till Dec. $15^{\text {th }}$. Significant differences were found among various wheat advance lines on days to heading, spike length, spike lets spike ${ }^{-1}$, grains spike-1, plant height, lodging score (\%), 1000-kernel weight, biological yield while flag leaf area, days to maturity, canopy temperature, and chlorophyll content were not significantly affected. The interactive effects among various wheat advance lines and different sowing dates were not significant except spike lets spike ${ }^{-1}$. Among various wheat advance lines PR-107 produced higher grain yield (4803kg ha-1) followed by PR-106 and PR-103 while minimum grain yield (3847 kg ha-1) were observed for PR-109 at all sowing dates. Among various sowing dates, Oct. $25^{\text {th }}$ sown crop resulted statistically maximum grain yield (5196kg ha $\left.{ }^{-1}\right)$ followed by Nov. $05^{\text {th }}$ sown crop $(5008 \mathrm{~kg}$ ha$\left.{ }^{1}\right)$ against minimum grain yield (3366kg ha-1) of Dec. $15^{\text {th }}$ sown crops. Average performance of wheat advance lines PR-107 and PR-103 on Oct. $25^{\text {th }}$ and Nov. $5^{\text {th }}$ sowing dates, were recorded $\left(5537 \mathrm{~kg} \mathrm{ha}^{-1}\right)$ and $\left(5287 \mathrm{~kg} \mathrm{ha}^{-1}\right)$ respectively. Greater average yield of PR-106 $(5373 \mathrm{~kg}$ ha-1 $)$ were recorded on Nov. $15^{\text {th }}$ and Nov. $25^{\text {th }}$ sowing dates. Thus, these results indicates that wheat advance lines should be sown on optimum sowing time Oct $25^{\text {th }}$ and Nov. $05^{\text {th }}$ for producing maximum grain yield in the central agro-ecological zone of Khyber Pukhtunkhwa (KP).
\end{abstract}

Keywords: Wheat (Triticum aestivum L) advance lines; Planting dates; Agronomic traits; Grain yield

\section{Introduction}

Wheat (Triticum aestivum L) belongs to poaceae family, tribe hardene and genus triticum. In Pakistan it is called kanak (punjabi), ghanum (pushto), and gandum (urdu). Wheat is an annual, self pollinated and photo periodically long day plant. Wheat has four main parts including root, stem, leaves and inflorescence (spike). Wheat three groups diploids had 14(n=7), tetraploids had 28(n=14) and the hexaploids had 42(n=21) chromosomes. Wheat grain contains all essential nutrients; kernel contains about 12 percent water, including carbohydrates
(60-80\% mainly as starch), proteins (8-15\%) containing adequate amounts of all essential amino acids (except lysine, tryptophan and methionine), fats (1.5-2\%), wheat is adapted to temperate regions from $30-60^{\circ} \mathrm{N}$ and $27-40{ }^{\circ} \mathrm{S}$ latitudes.

Wheat is most important cereal crop for the majority of world's populations. It is the most important staple food of about two million people (36\% of the world population) today wheat is grown all over the world, with different varieties sown according to the various climates the greatest portion of the wheat flour produced is used for bread making. Winter and spring wheat's 
are the two major types of the crop, with the severity of the winter determining whether a winter or spring type is cultivated.

It is important food crop of Pakistan grown during the winter season. It is being eaten through out the country daily three times. Wheat crop occupies a central position in forming agricultural policies and dominates all crops in acreage and production. Demand for wheat in the country is increasing day by day. The greatest demand for wheat in the coming years will have to be met by increasing devotion of land to wheat and or increasing yield per unit area. In 2012-13, wheat grown in Pakistan on an area of 8693 thousand hectare with total production of 24231 thousand tons, making an average yield of 2786kg per hectare (Pakistan economic survey 2012-13).

Low yield of wheat in Khyber Pakhtunkhwa and Pakistan as a whole is attributed to various factors such as planting date is one of the major factors which determine the ability of the crop to stand against different environmental conditions (air, temperature and humidity). Appropriate sowing date is important to have the crop in the field, when environmental conditions are conducive for growth and development. Variation in weather conditions among and within seasons is one of the most important constraints affecting yield potential [1]. Therefore one of the requirements for obtaining high yield is the choice of the suitable sowing date due to variations in weather conditions among seasons and developing new high yielding cultivars and by adopting proper sowing date. One of the most important factors influencing the wheat yield is sowing time. Many researchers have carried out some studies on sowing times of wheat and found different results [2,3].

Wheat is sown in winter and it has its own definite requirements for temperature and light for emergence, growth and flowering [4]. Early sowing always produces higher yield than late sowing. Early sown wheat had higher grain yield Qamar et al. [5]. The detrimental effect of delayed sowing on grain yield was maximum with reduction in 1000-kernel weight [5,6].

Delayed sowing also significantly reduced test weight [7]. High temperature and desiccating winds during the month of April might cause forced maturity of late sown wheat, thus resulting in reduction of test weight [8]. Higher grain crude protein content but lower grain sizes were obtained with delayed sowing [9]. High temperature in the post anthesis period of late sown wheat shortened the grain filling period resulting in a smaller endosperm, lower grain weight and increased protein content [10]. Subhan et al. [11] and Qasim et al. [12] concluded that crop planted on November 15, produced higher grain yield as compared to late and early planting. However, midseason sowing of winter wheat for any locality is usually most favourable. When optimum condition was provided by the wheat cultivar, grain filling period was higher as compared to late sown condition under high temperature stress at maturity.

Cereal Crops Research Institute (CCRI), Pirsabak Nowshera (KPK) Pakistan has the mandate to produce wheat varieties suitable for normal and late planting in rainfed and irrigated areas of the province. Every year, the promising lines are tested in a sowing dates trial to record their yield performance in various sowing dates and later on make recommendations for these prospective wheat varieties for normal and/or late planting in various parts of KP. The present project is therefore, designed with the objectives to find out the performance of different wheat (Triticum aestivem L.) advance lines (PR-103, PR-105, PR106, PR-107, PR-108 and PR-109) for agronomic traits and grain yield under different sowing dates (Oct. $25^{\text {th }}$, Nov. $5^{\text {th }}$, Nov. $15^{\text {th }}$, Nov. $25^{\text {th }}$, Dec. $5^{\text {th }}$ and Dec. $15^{\text {th }}$.

\section{Materials and Methods}

The experiment was conducted at Cereal Crops Research Institute, Pirsabak Nowshera, Khyber Pakhtunkhwa, Pakistan during 2013-14. The experimental site located at $32{ }^{\circ} \mathrm{N}$ Latitude, $74^{\circ} \mathrm{E}$ Longitude and $288 \mathrm{~m}$ Altitude. The soil type of experimental site was sandy loam having a pH range from 7 to 8 . Six newly developed wheat advance lines (PR-103, PR-105, PR106, PR-107, PR-108 and PR-109) were planted on six different sowing dates (Oct. $25^{\text {th }}$, Nov. $5^{\text {th }}$, Nov. $15^{\text {th }}$, Nov. $25^{\text {th }}$, Dec. $5^{\text {th }}$ and Dec. $\left.15^{\text {th }}\right)$ in a randomized complete block design with split plot arrangement repeated thrice. Each entry was grown in six rows of five meters long with row to row distance of $30 \mathrm{~cm}$. Plot area was $9 \mathrm{~m}^{2}$. Uniform seed rate of $100 \mathrm{~kg}$ per hectare was used. Planting was done with hand hoe. Fertilizer was applied at the ratio of 120:60kg per hectare of N:P in the form of Urea and Single Super Phosphate (SSP). SSP was applied as basal dose at the time of sowing while urea was applied in split doses; half at the time of sowing and half with first irrigation. All other recommended and standard agronomic practices were kept normal and uniform for all the experimental units in order to reduce experimental error. Data were recorded from central four rows in each subplot.

\section{Statistical Analysis}

Data were analyzed statistically with the statistical package MSTAT-C (Knowledge Dynamics Corporation, USA). The ANOVA test was used to determine significant $(p \leq 0.01$ or $p \leq 0.05)$ treatment effect. LSD test was also applied for the significance of treatment differences.

\section{Results and Discussion}

\section{Days to $50 \%$ heading}

Mean results (Table 1) showed that significant differences for days to $50 \%$ heading of various wheat advance lines sown at different dates. Maximum number of days to $50 \%$ heading (130 days) were recorded for Oct. $25^{\text {th }}$ sown crop. Days to $50 \%$ heading decreased gradually (107 days) as sowing was delayed from Oct. $25^{\text {th }}$ till Dec. $15^{\text {th }}$. Similar finding were also reported by Khan et al. [14] stated that growing crops at different sowing dates pass through each development stage under different environmental conditions. There was no significant difference of days to $50 \%$ heading among wheat lines except PR-109, took minimum days to heading as compared to PR-107 and PR-106. 
However, as the time passed, differences between wheat advance lines became prominent. Minimum number of days $(107,107$ and 108 days) was recorded for wheat lines PR- 103, PR-105 and PR-106 showing decrease 18.3, 17 and 19.4\% respectively while PR-107, PR-108 and PR-109 took 108, 106 and 105 days, showing decrease $19,15.8$ and $16 \%$ respectively in days to $50 \%$ heading, when wheat lines were sown on Oct. $25^{\text {th }}$ as compared to Dec. $15^{\text {th }}$ (Table 1). Wheat is sown in winter and it has its own definite requirements for temperature and light for emergence, growth and days to $50 \%$ heading.

Table 1: Mean values for days to $50 \%$ heading of newly developed wheat advance lines planted on different sowing dates evaluated at CCRI Pirsabak during 2013-2014.

\begin{tabular}{|c|c|c|c|c|c|c|c|}
\hline \multicolumn{7}{|c|}{ Sowing Dates } \\
\hline Wheat Lines & Oct. 25 & Nov. 5 & Nov. 15 & Nov. 25 & Dec. 5 & Dec.15 & Mean \\
\hline PR-103 & $131 \mathrm{ab}$ & $131 \mathrm{ab}$ & $122 \mathrm{~d}-\mathrm{h}$ & $119 \mathrm{~h}-\mathrm{k}$ & $114 \mathrm{i}-\mathrm{m}$ & $107 \mathrm{p}$ & $121 \mathrm{~b}$ \\
\hline PR-105 & $129 \mathrm{bc}$ & $130 \mathrm{bc}$ & $122 \mathrm{e}-\mathrm{h}$ & $117 \mathrm{i}-\mathrm{l}$ & $116 \mathrm{k}-\mathrm{m}$ & $107 \mathrm{p}$ & $120 \mathrm{bc}$ \\
\hline PR-106 & $134 \mathrm{a}$ & $132 \mathrm{ab}$ & $124 \mathrm{~d}-\mathrm{g}$ & $120 \mathrm{~g}-\mathrm{j}$ & $113 \mathrm{mn}$ & $108 \mathrm{p}$ & $122 \mathrm{a}$ \\
\hline PR-107 & $134 \mathrm{a}$ & $133 \mathrm{a}$ & $125 \mathrm{~d}-\mathrm{f}$ & $121 \mathrm{~g}-\mathrm{i}$ & $114 \mathrm{l}-\mathrm{m}$ & $108 \mathrm{p}$ & $123 \mathrm{a}$ \\
\hline PR-108 & $126 \mathrm{~cd}$ & $131 \mathrm{ab}$ & $121 \mathrm{f}-\mathrm{h}$ & $117 \mathrm{j}-\mathrm{m}$ & $114 \mathrm{i}-\mathrm{m}$ & $106 \mathrm{p}$ & $119 \mathrm{c}$ \\
\hline PR-109 & $125 \mathrm{de}$ & $124 \mathrm{~d}-\mathrm{g}$ & $117 \mathrm{j}-\mathrm{m}$ & $116 \mathrm{k}-\mathrm{m}$ & $111 \mathrm{no}$ & $105 \mathrm{p}$ & $116 \mathrm{~d}$ \\
\hline Mean & $130 \mathrm{a}$ & $130 \mathrm{a}$ & $122 \mathrm{~b}$ & $118 \mathrm{c}$ & $114 \mathrm{~d}$ & $107 \mathrm{e}$ & \\
\hline
\end{tabular}

Means in the same category following by different letters are significantly different from each other at $5 \%$ level of probability. LSD (sowing dates): 1.96 LSD (wheat advance lines): 1.40 LSD (sowing dates $x$ wheat advance lines): 3.43 .

\section{Days to physiological maturity}

Table 2: Mean values for days to physiological maturity of developed wheat advance lines planted on different sowing dates evaluated at CCRI Pirsabak during 2013-2014.

\begin{tabular}{|c|c|c|c|c|c|c|c|}
\hline \multicolumn{7}{|c|}{ Sowing Dates } \\
\hline Wheat Lines & Nov. 25 & Nov. 5 & Nov. 15 & Nov. 25 & Dec. 5 & Dec.15 & Mean \\
\hline PR-103 & $175 \mathrm{a}$ & $171 \mathrm{~d}-\mathrm{e}$ & $166 \mathrm{fg}$ & $159 \mathrm{hi}$ & $149 \mathrm{j}$ & $142 \mathrm{k}$ & $160.3 \mathrm{ab}$ \\
\hline PR-105 & $174 \mathrm{a}-\mathrm{c}$ & $172 \mathrm{~b}-\mathrm{e}$ & $167 \mathrm{f}$ & $159 \mathrm{hi}$ & $149 \mathrm{j}$ & $140 \mathrm{kl}$ & $160.3 \mathrm{ab}$ \\
\hline PR-106 & $174 \mathrm{a}-\mathrm{c}$ & $172 \mathrm{c}-\mathrm{e}$ & $166 \mathrm{fg}$ & $158 \mathrm{i}$ & $149 \mathrm{j}$ & $141 \mathrm{kl}$ & $160.1 \mathrm{ab}$ \\
\hline PR-107 & $175 \mathrm{ab}$ & $174 \mathrm{a}-\mathrm{c}$ & $164 \mathrm{~g}$ & $160 \mathrm{~h}$ & $149 \mathrm{j}$ & $142 \mathrm{k}$ & $160.7 \mathrm{a}$ \\
\hline PR-108 & $172 \mathrm{c}-\mathrm{e}$ & $171 \mathrm{e}$ & $167 \mathrm{f}$ & $159 \mathrm{hi}$ & $148 \mathrm{j}$ & $140 \mathrm{kl}$ & $159.6 \mathrm{~b}$ \\
\hline PR-109 & $174 \mathrm{a}-\mathrm{c}$ & $172 \mathrm{c}-\mathrm{e}$ & $167 \mathrm{f}$ & $158 \mathrm{hi}$ & $149 \mathrm{j}$ & $139 \mathrm{l}$ & $159.9 \mathrm{ab}$ \\
\hline Mean & $174 \mathrm{a}$ & $172 \mathrm{~b}$ & $166 \mathrm{c}$ & $159 \mathrm{~d}$ & $149 \mathrm{e}$ & $141 \mathrm{f}$ & \\
\hline
\end{tabular}

Means in the same category following by different letters are significantly different from each other at $5 \%$ level of probability. LSD (sowing dates): 1.05 LSD (wheat advance lines): 0.97 LSD (sowing dates $x$ wheat advance lines): 2.38 .

Mean results (Table 2) revealed that significant affect on days to physiological maturity of various wheat advance lines sown at different dates. Maximum number of days to maturity (174 days) was recorded for Oct. $25^{\text {th }}$ sown crop. Days to maturity decreased (141 days) gradually as sowing was delayed till Dec. $5^{\text {th }}$. Subhan et al. [15] also reported decrease in days to maturity due to delay in sowing in the central agro-ecological zone of KP Pakistan. There was no significant difference of days to physiological maturity among various wheat advance lines. However, as the time passed, differences between wheat advance lines became prominent. Minimum number of days 142, 140 and 141 were recorded for wheat lines PR-103, PR-105, and PR-106 showing decrease 18.8, 19.5 and $18.9 \%$ respectively while PR-107, PR108 and PR-109 were recorded 142, 140 and 139 days showing decrease $18.8,18.6$ and $19.4 \%$ respectively in days to maturity, when wheat lines were sown on Oct. $25^{\text {th }}$ as compared to Dec. $15^{\text {th }}$ (Table 2). Such wheat lines, if high yielding, are the most suitable for KP zone.

\section{Canopy temperature at post anthesis stage $\left({ }^{\circ} \mathrm{C}\right)$}

Mean results (Table 3) revealed that significant affect on canopy temperature at post anthesis stage of various wheat advance lines sown at different dates. Maximum canopy temperature at post anthesis stage $\left(24.4{ }^{\circ} \mathrm{C}\right)$ was recorded for Dec. $15^{\text {th }}$ sown crop while minimum $\left(21^{\circ} \mathrm{C}\right)$ was recorded for Nov. $5^{\text {th }}$ sown crop. There was no significant difference of canopy temperature at post anthesis stage among various wheat advance lines. Differences between wheat advance lines became 
prominent with passage of time. Mean canopy temperature (21.4 ${ }^{\circ} \mathrm{C}$ ) at post anthesis stage was recorded for Oct. sown crop in wheat lines PR-103, PR-105, and PR-106 showing increase 11.4,
11.2 and $10 \%$ respectively while $\left(21.4{ }^{\circ} \mathrm{C}\right)$ was recorded for PR107, PR-108 and PR-109 showing increase 11.6, 11.3 and 11.1\% respectively over Dec. $15^{\text {th }}$ sown crop.

Table 3: Mean values for canopy temperature $\left({ }^{\circ} \mathrm{C}\right)$ at post anthesis stage of newly developed wheat advance lines planted on different sowing dates evaluated at CCRI Pirsabak during 2013-2014.

\begin{tabular}{|c|c|c|c|c|c|c|c|}
\hline \multicolumn{9}{|c|}{ Sowing Dates } \\
\hline Wheat Lines & Oct. 25 & Nov. 5 & Nov. 15 & Nov. 25 & Dec. 5 & Dec.15 & Mean \\
\hline PR-103 & $21.6 \mathrm{e}-\mathrm{i}$ & $21.3 \mathrm{f}-\mathrm{j}$ & $21.0 \mathrm{~h}-\mathrm{j}$ & $22.3 \mathrm{~d}-\mathrm{h}$ & $24.4 \mathrm{a}$ & $24.4 \mathrm{a}$ & $22.5 \mathrm{a}$ \\
\hline PR-105 & $21.4 \mathrm{f}-\mathrm{j}$ & $21.1 \mathrm{~h}-\mathrm{j}$ & $22.2 \mathrm{~d}-\mathrm{h}$ & $22.4 \mathrm{~d}-\mathrm{h}$ & $24.4 \mathrm{a}$ & $24.8 \mathrm{a}$ & $22.7 \mathrm{a}$ \\
\hline PR-106 & $21.2 \mathrm{f}-\mathrm{j}$ & $20.1 \mathrm{j}$ & $21.8 \mathrm{e}-\mathrm{i}$ & $23.4 \mathrm{a}-\mathrm{d}$ & $24.1 \mathrm{ab}$ & $24.2 \mathrm{ab}$ & $22.5 \mathrm{a}$ \\
\hline PR-107 & $21.8 \mathrm{e}-\mathrm{i}$ & $21.0 \mathrm{~h}-\mathrm{j}$ & $22.5 \mathrm{~d}-\mathrm{g}$ & $22.6 \mathrm{c}-\mathrm{f}$ & $24.0 \mathrm{a}-\mathrm{c}$ & $24.5 \mathrm{a}$ & $22.8 \mathrm{a}$ \\
\hline PR-108 & $21.4 \mathrm{f}-\mathrm{j}$ & $20.7 \mathrm{i}-\mathrm{j}$ & $21.7 \mathrm{e}-\mathrm{i}$ & $21.4 \mathrm{f}-\mathrm{j}$ & $24.3 \mathrm{a}$ & $24.1 \mathrm{ab}$ & $22.3 \mathrm{a}$ \\
\hline PR-109 & $21.2 \mathrm{f}-\mathrm{j}$ & $21.8 \mathrm{e}-\mathrm{i}$ & $21.1 \mathrm{~h}-\mathrm{j}$ & $22.9 \mathrm{~b}-\mathrm{e}$ & $23.5 \mathrm{a}-\mathrm{d}$ & $24.5 \mathrm{a}$ & $22.5 \mathrm{a}$ \\
\hline Mean & $21.4 \mathrm{~cd}$ & $21.0 \mathrm{~d}$ & $21.7 \mathrm{c}$ & $22.5 \mathrm{~b}$ & $24.1 \mathrm{a}$ & $24.4 \mathrm{a}$ & \\
\hline
\end{tabular}

Means in the same category following by different letters are significantly different from each other at $5 \%$ level of probability. LSD (sowing dates): 0.67 LSD (wheat advance lines): 0.57 LSD (sowing dates $x$ wheat advance lines): 1.41.

\section{Chlorophyll content at post anthesis stage (mg)}

Table 4: Mean values for chlorophyll content $(\mathrm{mg})$ at post anthesis stage of newly developed wheat advance lines planted on different sowing dates evaluated at CCRI Pirsabak during 2013-2014.

\begin{tabular}{|c|c|c|c|c|c|c|c|}
\hline \multicolumn{9}{|c|}{ Sowing Dates } \\
\hline Wheat Lines & Oct. 25 & Nov. 5 & Nov. 15 & Nov. 25 & Dec. 5 & Dec.15 & Mean \\
\hline PR-103 & $44.5 \mathrm{a}-\mathrm{e}$ & $43.9 \mathrm{a}-\mathrm{f}$ & $46.2 \mathrm{ab}$ & $41.9 \mathrm{a}-\mathrm{g}$ & $42.7 \mathrm{a}-\mathrm{g}$ & $37.7 \mathrm{~g}$ & $42.8 \mathrm{a}$ \\
\hline PR-105 & $42.6 \mathrm{a}-\mathrm{g}$ & $43.5 \mathrm{a}-\mathrm{g}$ & $42.6 \mathrm{a}-\mathrm{g}$ & $41.4 \mathrm{a}-\mathrm{g}$ & $41.3 \mathrm{a}-\mathrm{g}$ & $39.5 \mathrm{~d}-\mathrm{g}$ & $41.7 \mathrm{a}$ \\
\hline PR-106 & $47.2 \mathrm{a}$ & $44.1 \mathrm{a}-\mathrm{f}$ & $41.4 \mathrm{a}-\mathrm{g}$ & $40.3 \mathrm{~b}-\mathrm{g}$ & $44.5 \mathrm{a}-\mathrm{e}$ & $38.1 \mathrm{fg}$ & $42.6 \mathrm{a}$ \\
\hline PR-107 & $44.9 \mathrm{a}-\mathrm{d}$ & $43.2 \mathrm{a}-\mathrm{g}$ & $40.9 \mathrm{~b}-\mathrm{g}$ & $41.0 \mathrm{~b}-\mathrm{g}$ & $42.6 \mathrm{a}-\mathrm{g}$ & $38.2 \mathrm{fg}$ & $41.1 \mathrm{a}$ \\
\hline PR-108 & $43.4-\mathrm{gg}$ & $44.0 \mathrm{a}-\mathrm{f}$ & $41.6 \mathrm{a}-\mathrm{g}$ & $41.2 \mathrm{a}-\mathrm{g}$ & $40.7 \mathrm{~b}-\mathrm{g}$ & $40.1 \mathrm{~b}-\mathrm{g}$ & $41.8 \mathrm{a}$ \\
\hline PR-109 & $45.1 \mathrm{a}-\mathrm{c}$ & $38.5 \mathrm{e}-\mathrm{g}$ & $39.4 \mathrm{e}-\mathrm{g}$ & $43.1 \mathrm{a}-\mathrm{g}$ & $40.4 \mathrm{~b}-\mathrm{g}$ & $42.8 \mathrm{a}-\mathrm{g}$ & $41.5 \mathrm{a}$ \\
\hline Mean & $44.6 \mathrm{a}$ & $42.8 \mathrm{ab}$ & $42.0 \mathrm{~b}$ & $41.5 \mathrm{~b}$ & $42.1 \mathrm{~b}$ & $39.0 \mathrm{c}$ & \\
\hline
\end{tabular}

Means in the same category following by different letters are significantly different from each other at $5 \%$ level of probability. LSD (sowing dates): 1.77 LSD (wheat advance lines): 2.59 LSD (sowing dates $x$ wheat advance lines): 6.12.

After computing mean results (Table 4) significant affect on chlorophyll content at post anthesis of various wheat advance lines sown at different dates. Maximum chlorophyll content at post anthesis stage $(44.6 \mathrm{mg})$ was recorded for Oct. $25^{\text {th }}$ sown crop and minimum chlorophyll content $(39 \mathrm{mg})$ was recorded for Dec. $15^{\text {th }}$ sown crop. There was no significant difference of chlorophyll content at post anthesis stage among various wheat advance lines. Differences between wheat advance lines became prominent with passage of time. Chlorophyll content 44.5, 42.6 and $47.2 \mathrm{mg}$ at post anthesis stage was recorded for Oct. sown crop in wheat lines PR- 103, PR-105 and PR-106 showing 15.2, 7.2 and $18.2 \%$ decrease respectively while $44.9,43.4$ and 45.1 mg was recorded for PR-107, PR-108 and PR-109 showing 14.9, 7.6 and $5 \%$ decrease respectively over Dec. $15^{\text {th }}$ sown crop.

\section{Flag Leaf Area $\left(\mathrm{Cm}^{2}\right)$}

Mean results (Table 5) showed that flag leaf area was significantly decreased as planting delay. There were also significant differences among wheat lines for flag leaf area. Maximum flag leaf area $\left(39.9 \mathrm{~cm}^{2}\right)$ was recorded for Nov. $5^{\text {th }}$ sown crop across all sowing dates, while significantly minimum flag leaf area $(30 \mathrm{~cm})$ was recorded for Dec. $15^{\text {th }}$ sown crop due delayed planting. Maximum flag leaf area $\left(37.3 \mathrm{~cm}^{2}\right)$ was recorded for wheat line PR-108 while minimum flag leaf area $(33.8 \mathrm{~cm})$ was recorded for PR-107 at all sowing date. Flag leaf area was decreased from 9 to $20 \%$ respectively when planting delayed from Oct. $25^{\text {th }}$ to Dec $15^{\text {th }}$. Elevation in temperature accelerates plant development while declined growth rate showed decline 
in leaf size and spike size which ultimately resulted in low yield [16]. The significant interaction between genotype and sowing

dates shows the sensitivity of different genotypes to photoperiod and temperature differently for flag leaf area.

Table 5: Mean values for flag leaf area $\left(\mathrm{cm}^{2}\right)$ of newly developed wheat advance lines planted on different sowing dates evaluated at CCRI Pirsabak during $2013-14$.

\begin{tabular}{|c|c|c|c|c|c|c|c|}
\hline \multicolumn{9}{|c|}{ Sowing Dates } \\
\hline Wheat Lines & Oct. 25 & Nov. 5 & Nov. 15 & Nov. 25 & Dec. 5 & Dec.15 & Mean \\
\hline PR103 & $39.5 \mathrm{a}-\mathrm{c}$ & $39.4 \mathrm{a}-\mathrm{d}$ & $35.2 \mathrm{~b}-\mathrm{h}$ & $34.1 \mathrm{c}-\mathrm{j}$ & $30.2 \mathrm{~h}-\mathrm{k}$ & $30.4 \mathrm{~h}-\mathrm{k}$ & $34.8 \mathrm{~b}$ \\
\hline PR105 & $37.9 \mathrm{a}-\mathrm{e}$ & $39.3 \mathrm{a}-\mathrm{d}$ & $41.0 \mathrm{a}$ & $36.5 \mathrm{a}-\mathrm{g}$ & $32.3 \mathrm{f}-\mathrm{k}$ & $28.6 \mathrm{jk}$ & $35.9 \mathrm{ab}$ \\
\hline PR106 & $33.9 \mathrm{~d}-\mathrm{k}$ & $39.0 \mathrm{a}-\mathrm{d}$ & $34.1 \mathrm{c}-\mathrm{j}$ & $34.8 \mathrm{~b}-\mathrm{i}$ & $30.0 \mathrm{~h}-\mathrm{k}$ & $30.8 \mathrm{~h}-\mathrm{k}$ & $33.8 \mathrm{~b}$ \\
\hline PR107 & $35.3 \mathrm{~b}-\mathrm{h}$ & $41.2 \mathrm{a}$ & $37.0 \mathrm{a}-\mathrm{g}$ & $37.5 \mathrm{a}-\mathrm{f}$ & $29.4 \mathrm{i}-\mathrm{k}$ & $30.2 \mathrm{~h}-\mathrm{k}$ & $35.1 \mathrm{ab}$ \\
\hline PR108 & $40.0 \mathrm{ab}$ & $41.3 \mathrm{a}$ & $41.2 \mathrm{a}$ & $39.1 \mathrm{a}-\mathrm{d}$ & $30.8 \mathrm{~h}-\mathrm{k}$ & $31.4 \mathrm{~g}-\mathrm{k}$ & $37.3 \mathrm{a}$ \\
\hline PR109 & $34.7 \mathrm{~b}-\mathrm{i}$ & $38.8 \mathrm{a}-\mathrm{d}$ & $39.0 \mathrm{a}-\mathrm{d}$ & $35.2 \mathrm{~b}-\mathrm{h}$ & $32.8 \mathrm{e}-\mathrm{k}$ & $3 . \mathrm{k}$ & $34.8 \mathrm{~b}$ \\
\hline Mean & $36.9 \mathrm{~b}$ & $39.9 \mathrm{a}$ & $37.9 \mathrm{ab}$ & $36.2 \mathrm{~b}$ & $30.9 \mathrm{c}$ & $30.0 \mathrm{c}$ & \\
\hline
\end{tabular}

Means in the same category following by different letters are significantly different from each other at $5 \%$ level of probability. LSD (sowing dates): 2.60 LSD (wheat advance lines): 2.22 LSD (sowing dates $x$ wheat advance lines): 5.44 .

\section{Spike Length $(\mathrm{Cm})$}

Table 6: Mean values for spike length $(\mathrm{cm})$ of newly developed wheat advance lines planted on different sowing dates evaluated at CCRI Pirsabak during 2013-2014.

\begin{tabular}{|c|c|c|c|c|c|c|c|}
\hline \multicolumn{9}{|c|}{ Sowing Dates } \\
\hline Wheat Lines & Oct. 25 & Nov. 5 & Nov. 15 & Nov. 25 & Dec. 5 & Dec.15 & Mean \\
\hline PR-103 & $10.8 \mathrm{~g}-\mathrm{j}$ & $10.8 \mathrm{~g}-\mathrm{j}$ & $10.3 \mathrm{~d}-\mathrm{h}$ & $12.7 \mathrm{ab}$ & $10.9 \mathrm{~g}-\mathrm{j}$ & $10.4 \mathrm{c}-\mathrm{f}$ & $11.1 \mathrm{a}$ \\
\hline PR-105 & $10.0 \mathrm{~d}$ & $9.7 \mathrm{~h}-\mathrm{m}$ & $10.4 \mathrm{~d}-\mathrm{h}$ & $12.4 \mathrm{ab}$ & $10.4 \mathrm{~d}-\mathrm{h}$ & $9.9 \mathrm{~h}-\mathrm{m}$ & $10.4 \mathrm{~b}$ \\
\hline PR-106 & $9.3 \mathrm{n}$ & $9.8 \mathrm{~h}-\mathrm{m}$ & $10.2 \mathrm{~d}-\mathrm{h}$ & $10.8 \mathrm{~g}-\mathrm{j}$ & $10.0 \mathrm{~d}$ & $9.3 \mathrm{n}$ & $9.9 \mathrm{~b}$ \\
\hline PR-107 & $10.5 \mathrm{c}-\mathrm{f}$ & $10.4 \mathrm{~d}-\mathrm{h}$ & $9.8 \mathrm{~h}-\mathrm{m}$ & $11.3 \mathrm{ab}$ & $10.3 \mathrm{~d}-\mathrm{h}$ & $9.3 \mathrm{n}$ & $10.3 \mathrm{~b}$ \\
\hline PR-108 & $10.6 \mathrm{c}-\mathrm{f}$ & $11.0 \mathrm{a}-\mathrm{c}$ & $10.6 \mathrm{c}-\mathrm{f}$ & $13.0 \mathrm{a}$ & $10.5 \mathrm{c}-\mathrm{f}$ & $9.8 \mathrm{~h}-\mathrm{m}$ & $10.9 \mathrm{~b}$ \\
\hline PR-109 & $9.4 \mathrm{i}-\mathrm{n}$ & $10.2 \mathrm{~d}-\mathrm{h}$ & $10.2 \mathrm{~d}-\mathrm{h}$ & $11.8 \mathrm{ab}$ & $9.8 \mathrm{~h}-\mathrm{m}$ & $10.1 \mathrm{~d}$ & $10.2 \mathrm{~b}$ \\
\hline Mean & $10.8 \mathrm{a}$ & $10.3 \mathrm{bc}$ & $10.2 \mathrm{bc}$ & $12.0 \mathrm{a}$ & $10.8 \mathrm{a}$ & $9.7 \mathrm{c}$ & \\
\hline
\end{tabular}

Means in the same category following by different letters are significantly different from each other at $5 \%$ level of probability. LSD (sowing dates): 0.52 LSD (wheat advance lines): 0.37 LSD (sowing dates $x$ wheat advance lines): 0.91.

Mean results (Table 6) revealed that significant differences for spike length of various wheat advance lines sown at different dates. Spike length was significantly decreased from $10.8 \mathrm{~cm}$ to $9.7 \mathrm{~cm}$ when planting delayed from Oct. $25^{\text {th }}$ to Dec. $15^{\text {th }}$. Maximum spike length $(11.1 \mathrm{~cm})$ was produced for wheat line PR-103 while minimum spikes length $(9.9 \mathrm{~cm})$ was produced for PR-106 at all sowing dates. Spike length ranged from $9.3 \mathrm{~cm}$ to $10.8 \mathrm{~cm}$ for all genotypes decreased from 3 to $7 \%$ respectively when wheat lines were sown from Oct. $25^{\text {th }}$ to delay till Dec. $15^{\text {th }}$ There was no significant difference of spike length among various wheat advance lines except PR-106 which have $9.9 \mathrm{~cm}$ spike length as compare to PR-103 $(11.1 \mathrm{~cm})$ at all sowing dates. The spike length probably decreased due to delay in sowing because of the sensitivity of the wheat plants to photoperiod and temperature [17]. As the photoperiod and temperature increased, the wheat plants hastened maturity to complete growth period due to which the plant parameters, like plant height and spike length got shorter period to increase in size. Slafer [18] have suggested that there is a strong interaction between the responses to temperature and photoperiod in terms of various plant parameters and that the size of interaction may different among genotypes. The significant interaction between genotype and sowing dates for spike length signifies the sensitivity of different genotypes to photoperiod and temperature differently and, thus, confirms the suggestions of Slafer [18].

\section{Spikelets Spike-1}

Mean results (Table 7) showed that significant differences for spikelets spike ${ }^{-1}$ of various wheat advance lines sown at different dates. Spikelets spike ${ }^{-1}$ was significantly decreased from 17.2 to (15.9) when planting was delayed from Oct. $25^{\text {th }}$ to Dec. 
$15^{\text {th }}$. Maximum number of spikelets spike ${ }^{-1}(18.8)$ was produce for wheat line PR-103 which was not significantly different from that of PR-107 and PR-105, while minimum number of spikelets spike ${ }^{-1}$ (16.6) was produce for PR-109 at all sowing dates. The number of spikelets spike ${ }^{-1}$ probably decreased with delay in planting date because of reduction in spike length. The decrease was significant when planting was delayed from Oct. $25^{\text {th }}$ to Dec. $15^{\text {th }}$; it decreased to $7.5 \%$ respectively. According to Shafiq [19] early sowing enhanced spikelets spike ${ }^{-1}$ over late sowing. There was no significant difference of spikelets spike-1 among various wheat advance lines except PR-109 which have less number (16.6) and significantly effected as compare to other wheat advance lines. The number of spikelets spike ${ }^{-1}$ probably decreased because of reduction in spike length, although it is not necessary because in lax spikes, the number of spikelets spike ${ }^{-1}$ is small and it is the characteristic of some varieties [20].

Table 7: Mean values for spikelets spike ${ }^{-1}$ of newly developed wheat advance lines planted on different sowing dates evaluated at CCRI Pirsabak during 2013-2014.

\begin{tabular}{|c|c|c|c|c|c|c|c|}
\hline \multicolumn{7}{|c|}{ Sowing Dates } \\
\hline Wheat Lines & Oct. 25 & Nov. 5 & Nov. 15 & Nov. 25 & Dec. 5 & Dec.15 & Mean \\
\hline PR-103 & $16.7 \mathrm{e}-\mathrm{h}$ & $19.7 \mathrm{ab}$ & $19.0 \mathrm{a}-\mathrm{c}$ & $17.7 \mathrm{c}-\mathrm{g}$ & $16.7 \mathrm{e}-\mathrm{h}$ & $17.4 \mathrm{c}-\mathrm{h}$ & $18.8 \mathrm{ab}$ \\
\hline PR-105 & $18.7 \mathrm{~b}-\mathrm{d}$ & $18.3 \mathrm{~b}-\mathrm{e}$ & $18.0 \mathrm{~b}-\mathrm{f}$ & $18.0 \mathrm{~b}-\mathrm{e}$ & $17.3 \mathrm{c}-\mathrm{h}$ & $16.0 \mathrm{gh}$ & $17.7 \mathrm{ab}$ \\
\hline PR-106 & $17.0 \mathrm{c}-\mathrm{h}$ & $18.3 \mathrm{~b}-\mathrm{e}$ & $18.0 \mathrm{~b}-\mathrm{f}$ & $18.3 \mathrm{~b}-\mathrm{e}$ & $16.3 \mathrm{f}-\mathrm{h}$ & $16.0 \mathrm{gh}$ & $17.3 \mathrm{~b}$ \\
\hline PR-107 & $18.3 \mathrm{~b}-\mathrm{e}$ & $20.7 \mathrm{a}$ & $19.7 \mathrm{ab}$ & $17.7 \mathrm{c}-\mathrm{g}$ & $17.0 \mathrm{c}-\mathrm{h}$ & $17.0 \mathrm{c}-\mathrm{h}$ & $18.4 \mathrm{a}$ \\
\hline PR-108 & $16.3 \mathrm{f}-\mathrm{h}$ & $18.0 \mathrm{~b}-\mathrm{f}$ & $18.3 \mathrm{~b}-\mathrm{e}$ & $19.0 \mathrm{a}-\mathrm{c}$ & $16.3 \mathrm{f}-\mathrm{h}$ & $15.7 \mathrm{~h}$ & $17.3 \mathrm{~b}$ \\
\hline PR-109 & $16.6 \mathrm{e}-\mathrm{h}$ & $17.0 \mathrm{c}-\mathrm{h}$ & $17.7 \mathrm{c}-\mathrm{g}$ & $17.7 \mathrm{c}-\mathrm{g}$ & $16.7 \mathrm{e}-\mathrm{h}$ & $13.7 \mathrm{i}$ & $16.6 \mathrm{~b}$ \\
\hline Mean & $17.2 \mathrm{bc}$ & $18.7 \mathrm{a}$ & $18.4 \mathrm{a}$ & $18.1 \mathrm{bc}$ & $16.7 \mathrm{~cd}$ & $15.9 \mathrm{~d}$ & \\
\hline
\end{tabular}

Means in the same category following by different letters are significantly different from each other at $5 \%$ level of probability. LSD (sowing dates): 0.93 LSD (wheat advance lines): 0.68 LSD (sowing dates $x$ wheat advance lines): 1.67.

\section{Number of Grains Spike ${ }^{-1}$}

Table 8: Mean values for number of grains spike ${ }^{-1}$ of newly developed wheat advance lines planted on different sowing dates evaluated at CCRI Pirsabak during 2013-2014.

\begin{tabular}{|c|c|c|c|c|c|c|c|}
\hline \multicolumn{7}{|c|}{ Sowing Dates } \\
\hline Wheat Lines & Oct. 25 & Nov. 5 & Nov. 15 & Nov. 25 & Dec. 5 & Dec.15 & Mean \\
\hline PR-103 & $65.3 \mathrm{a}-\mathrm{d}$ & $66.7 \mathrm{a}-\mathrm{c}$ & $64.3 \mathrm{a}-\mathrm{e}$ & $65.7 \mathrm{a}-\mathrm{d}$ & $57.3 \mathrm{e}-\mathrm{f}$ & $56.0 \mathrm{f}-\mathrm{i}$ & $57.6 \mathrm{a}$ \\
\hline PR-105 & $66.7 \mathrm{a}-\mathrm{c}$ & $67.7 \mathrm{ab}$ & $71.0 \mathrm{a}$ & $63.7 \mathrm{~b}-\mathrm{e}$ & $59.0 \mathrm{~d}-\mathrm{e}$ & $55.0 \mathrm{~g}-\mathrm{i}$ & $57.4 \mathrm{a}$ \\
\hline PR-106 & $56.0 \mathrm{f}-\mathrm{i}$ & $61.3 \mathrm{c}-\mathrm{e}$ & $61.0 \mathrm{c}-\mathrm{e}$ & $64.0 \mathrm{a}-\mathrm{e}$ & $56.3 \mathrm{f}-\mathrm{i}$ & $55.0 \mathrm{~g}-\mathrm{i}$ & $56.3 \mathrm{ab}$ \\
\hline PR-107 & $59.7 \mathrm{~d}-\mathrm{e}$ & $68.0 \mathrm{ab}$ & $66.3 \mathrm{a}-\mathrm{c}$ & $63.0 \mathrm{~b}-\mathrm{e}$ & $62.3 \mathrm{~b}-\mathrm{e}$ & $52.2 \mathrm{hi}$ & $55.4 \mathrm{ab}$ \\
\hline PR-108 & $57.0 \mathrm{e}-\mathrm{f}$ & $67.3 \mathrm{ab}$ & $68.7 \mathrm{ab}$ & $65.7 \mathrm{a}-\mathrm{d}$ & $59.0 \mathrm{~d}-\mathrm{e}$ & $55.3 \mathrm{~g}-\mathrm{i}$ & $54.3 \mathrm{bc}$ \\
\hline PR-109 & $59.3 \mathrm{~d}-\mathrm{e}$ & $59.7 \mathrm{~d}-\mathrm{e}$ & $59.0 \mathrm{~d}-\mathrm{e}$ & $62.0 \mathrm{~b}-\mathrm{e}$ & $57.7 \mathrm{e}-\mathrm{f}$ & $58.7 \mathrm{i}$ & $53.3 \mathrm{c}$ \\
\hline Mean & $60.7 \mathrm{ab}$ & $65.1 \mathrm{a}$ & $65.1 \mathrm{a}$ & $64.0 \mathrm{a}$ & $58.6 \mathrm{ab}$ & $53.8 \mathrm{~b}$ & \\
\hline
\end{tabular}

Means in the same category following by different letters are significantly different from each other at $5 \%$ level of probability. LSD (sowing dates): 4.83 LSD (wheat advance lines): 3.44 LSD (sowing dates $x$ wheat advance lines): 8.44 .

Mean results (Table 8) showed that significant differences for grains spike ${ }^{-1}$ of various wheat advance lines sown at different dates. Number of grains spike ${ }^{-1}$ was significantly decreased from (60.7) to (53.8) when wheat lines were delayed from Oct. $25^{\text {th }}$ to Dec. $15^{\text {th }}$. Maximum number of grains spike ${ }^{-1}$ (65.3, 66.7, 59.7 and 59.3) was produced for wheat lines PR-103, PR-105, PR-107 and PR-109 showing decrease 14.8, 14.2, 17.5 and $17.8 \%$ respectively when planting was delayed from Oct. $25^{\text {th }}$ to Dec. $15^{\text {th }}$. There was no significant difference between PR106 and PR-108 at planting dates. These findings are supported by those of Hanson [21], who reported significant variation in number of grains spike ${ }^{-1}$ with sowing dates. Maximum number of grain spike ${ }^{-1}$ (57.6) was produced for wheat line PR-103 while minimum number grains spike ${ }^{-1}$ (53.3) was produced for PR-109 at all sowing dates. Less number of grains spike ${ }^{-1}$ in late sowing was due to less production of photosynthesis due to shorter growing period. These results are in line with those of Shahzad et al. [21]. Differences in number of grains spike ${ }^{-1}$ among wheat lines might be attributed to their genetic variability. These results are in line with those reported by Haider [22]. The grains spike ${ }^{-1}$ seemed to have decreased due to delay in sowing because of the sensitivity of the wheat plants to photoperiod and temperature. 


\section{Plant Height (Cm)}

Table 9: Mean values for plant height $(\mathrm{cm})$ of newly developed wheat advance lines planted on different sowing dates evaluated at CCRI Pirsabak during 2013-2014.

\begin{tabular}{|c|c|c|c|c|c|c|c|}
\hline \multicolumn{9}{|c|}{ Sowing Dates } \\
\hline Wheat Lines & Oct. 25 & Nov. 5 & Nov. 15 & Nov. 25 & Dec. 5 & Dec.15 & Mean \\
\hline PR-103 & $120 \mathrm{a}$ & $117 \mathrm{a}-\mathrm{c}$ & $115 \mathrm{a}-\mathrm{e}$ & $107 \mathrm{~g}-\mathrm{l}$ & $113 \mathrm{~b}-\mathrm{g}$ & $99 \mathrm{~m}-\mathrm{q}$ & $112 \mathrm{a}$ \\
\hline PR-105 & $115 \mathrm{a}-\mathrm{d}$ & $105 \mathrm{i}-\mathrm{o}$ & $108 \mathrm{e}-\mathrm{j}$ & $105 \mathrm{c}-\mathrm{i}$ & $192 \mathrm{j}-\mathrm{q}$ & $96 \mathrm{pq}$ & $105 \mathrm{~d}$ \\
\hline PR-106 & $118 \mathrm{ab}$ & $114 \mathrm{a}-\mathrm{f}$ & $114 \mathrm{~b}-\mathrm{g}$ & $111 \mathrm{c}-\mathrm{i}$ & $107 \mathrm{f}-\mathrm{l}$ & $98 \mathrm{o}-\mathrm{q}$ & $110 \mathrm{ab}$ \\
\hline PR-107 & $116 \mathrm{a}-\mathrm{d}$ & $110 \mathrm{c}-\mathrm{i}$ & $112 \mathrm{~b}-\mathrm{h}$ & $108 \mathrm{e}-\mathrm{j}$ & $105 \mathrm{~h}-\mathrm{n}$ & $101 \mathrm{k}-\mathrm{q}$ & $109 \mathrm{bc}$ \\
\hline PR-108 & $113 \mathrm{~b}-\mathrm{g}$ & $108 \mathrm{e}-\mathrm{j}$ & $107 \mathrm{~g}-\mathrm{l}$ & $109 \mathrm{~d}-\mathrm{j}$ & $103 \mathrm{j}-\mathrm{p}$ & $101-\mathrm{q}$ & $107 \mathrm{~cd}$ \\
\hline PR-109 & $111 \mathrm{c}-\mathrm{i}$ & $108 \mathrm{e}-\mathrm{j}$ & $106 \mathrm{~h}-\mathrm{m}$ & $99 \mathrm{n}-\mathrm{q}$ & $108 \mathrm{f}-\mathrm{k}$ & $97 \mathrm{q}$ & $105 \mathrm{~d}$ \\
\hline Mean & $116 \mathrm{a}$ & $110 \mathrm{~b}$ & $110 \mathrm{~b}$ & $107 \mathrm{c}$ & $107 \mathrm{c}$ & $99 \mathrm{~d}$ & \\
\hline
\end{tabular}

Means in the same category following by different letters are significantly different from each other at $5 \%$ level of probability. LSD (sowing dates): 3.02 LSD (wheat advance lines): 2.81 LSD (sowing dates $x$ wheat advance lines): 6.89 .

On average results (Table 9) revealed that plant height was significantly decreased as planting delay. There were also significant differences among the wheat advance lines for plant height. Maximum plant height $(116 \mathrm{~cm})$ was recorded for Oct sown crop across all sowing dates while minimum plant height $(99 \mathrm{~cm})$ was recorded over Dec. $15^{\text {th }}$ sown crop, which was significantly different. Maximum plant height $(112 \mathrm{~cm})$ was recorded for wheat lines PR-103 and minimum plant height $(105 \mathrm{~cm})$ was recorded for PR-109 at all sowing date. Plant height

\section{Lodging Score (\%)}

Table 10: Mean values for lodging score (\%) of newly developed wheat advance lines planted on different sowing dates evaluated at CCRI Pirsabak during 2013-2014.

\begin{tabular}{|c|c|c|c|c|c|c|c|}
\hline \multicolumn{8}{|c|}{ Sowing Dates } \\
\hline Wheat Lines & Oct. 25 & Nov. 5 & Nov. 15 & Nov. 25 & Dec. 5 & Dec.15 & Mean \\
\hline PR-103 & $7 c-d$ & $11 b-d$ & $7 c-d$ & $0 \mathrm{~d}$ & $0 \mathrm{~d}$ & $0 \mathrm{~d}$ & $4 \mathrm{bc}$ \\
\hline PR-105 & $1 \mathrm{~d}$ & $2 \mathrm{~d}$ & $5 \mathrm{~cd}$ & $0 \mathrm{~d}$ & $0 \mathrm{~d}$ & od & $1 \mathrm{c}$ \\
\hline PR-106 & $5 \mathrm{~cd}$ & $41 \mathrm{a}$ & $29 \mathrm{ab}$ & $2 d$ & $6 \mathrm{~cd}$ & $0 \mathrm{~d}$ & $14 \mathrm{a}$ \\
\hline PR-107 & $3 \mathrm{~d}$ & $25 \mathrm{a}-\mathrm{c}$ & $18 \mathrm{~b}-\mathrm{d}$ & $12 b-d$ & $1 \mathrm{~d}$ & $0 \mathrm{~d}$ & $10 \mathrm{ab}$ \\
\hline PR-108 & $1 \mathrm{~d}$ & $2 \mathrm{~d}$ & $7 \mathrm{c}-\mathrm{d}$ & $4 d$ & $0 \mathrm{~d}$ & $0 \mathrm{~d}$ & $2 c$ \\
\hline PR-109 & $0 \mathrm{~d}$ & $15 b-d$ & $5 \mathrm{~cd}$ & $0 \mathrm{~d}$ & $14 \mathrm{~b}-\mathrm{d}$ & $0 \mathrm{~d}$ & $6 \mathrm{bc}$ \\
\hline Mean & $3 a b$ & $16 a$ & $12 \mathrm{ab}$ & $3 a b$ & $4 a b$ & $0 \mathrm{~b}$ & \\
\hline
\end{tabular}

Means in the same category following by different letters are significantly different from each other at $5 \%$ level of probability. LSD (sowing dates): 13.47 LSD (wheat advance lines): 6.69 LSD (sowing dates $x$ wheat advance lines): 16.39.

Mean results (Table 10) showed that lodging score (\%) was not significantly decreased as planting delay. There were significant differences among the wheat advance lines for lodging score (\%). The maximum lodging score (16\%) was recorded for Nov. $05^{\text {th }}$ sown crop, this lodging score was highest across all sowing dates while minimum lodging score $(0 \%)$ was recorded for Dec. $15^{\text {th }}$ sown crop. The excessive rains and downpours in the growing season caused lodging on Nov. $05^{\text {th }}$. Maximum lodging score (14\%) was recorded for wheat line PR- was decreased from 11 to $19 \%$ respectively when wheat lines were delayed from Oct. $25^{\text {th }}$ to Dec $15^{\text {th }}$. Decrease in plant height in late sowing was due to shorter growing period. Early sown crop may have enjoyed the better environmental conditions especially the temperature and solar radiation which resulted to tallest plants. These results are in line with those reported by Shahzad et al. [21]. Differences in plant height among varieties might be attributed to their genetic diversity. These results are similar to those of Ahmad et al. [23].
106 and minimum lodging score (1\%) was recorded for PR-105 at all planting dates. Lodging score were decreased to $(0 \%)$ with delay in planting date.

\section{Biomass Yield $\left(\mathrm{Kg} \mathrm{Ha}^{-1}\right)$}

Mean results (Table 11) showed that biomass yield (kg ha $\left.{ }^{1}\right)$ was significantly decreased as planting delay. There were also significant differences among the wheat lines for biomass yield. Maximum biomass yield (19517kg ha-1) was recorded 
for Oct. $25^{\text {th }}$ sown crop across all sowing dates, while minimum biomass yield (9820 $\mathrm{kg} \mathrm{ha}^{-1}$ ) was recorded for Dec. $15^{\text {th }}$ sown crop, which was significantly different due to delay in sowing time. Among time of sowing, significant maximum biomass yield was recorded (19517 $\left.\mathrm{kg} \mathrm{ha}^{-1}\right)$ and (18548kg ha-1) for Oct $25^{\text {th }}$ and Nov. $05^{\text {th }}$ sown crop against minimum biomass yield $(9820 \mathrm{~kg}$ ha $\left.{ }^{1}\right)$ for Dec. $15^{\text {th }}$ sown crop. Average performance of wheat lines PR-107 and PR-106 were recorded (20100 kg ha-1) and (19646kg ha $^{-1}$ ) followed by PR-103 (18999 $\mathrm{kg} \mathrm{ha}^{-1}$ ), while PR-105 produce the lowest biomass yield (17998 $\left.\mathrm{kg} \mathrm{ha}^{-1}\right)$, when wheat lines were sown on Oct. $25^{\text {th }}$ and Nov. $5^{\text {th }}$. Maximum biomass yield $(16651 \mathrm{~kg}$ $\mathrm{ha}^{-1}$ ) was recorded for wheat lines PR-106 and followed by PR107 (15939 $\left.\mathrm{kg} \mathrm{ha}^{-1}\right)$, while minimum biomass yield $(14095 \mathrm{~kg}$ ha $\left.{ }^{1}\right)$ was recorded for PR-109 at all sowing dates. Biomass yield decreased gradually and significantly in all wheat advance lines with delay in sowing time. Yield losses due to late planting were recorded $4.9 \%$ when planting was done on Nov. $5^{\text {th }}, 11.1 \%$ when planting was done on Nov.15, 17.6\% when planting was done on Nov. $25^{\text {th }}, 25.4 \%$ when planting was done on Dec. $5^{\text {th }}$ and $49.6 \%$ when planting was further delayed till Dec. $15^{\text {th }}$ as compare to Oct. $25^{\text {th }}$. These findings are in agreements with those of Wajjid [24], who also reported that early planting increases biomass yield while late planting decreases biomass yield. Biomass yield decreased from 43.2 to $49.6 \%$ when wheat lines were delayed from Oct. $25^{\text {th }}$ to Dec. $25^{\text {th }}$. The first reason may be more suitable climatic condition for maximum vegetative and reproductive components resulted increased biomass yield. Secondly it may be due to reproductive plants unit per area. In later sowing date's biomass and grain yield decreased due to undesired environmental conditions and lack of suitable transforming preserved matters to seeds as a result of increasing temperature at the end of growth season.

Table 11: Mean values for biomass yield $\left(\mathrm{kg} \mathrm{ha}^{-1}\right)$ of newly developed wheat advance lines planted on different sowing dates evaluated at CCRI Pirsabak during 2013-2014.

\begin{tabular}{|c|c|c|c|c|c|c|c|}
\hline \multicolumn{7}{|c|}{ Sowing Dates } \\
\hline Wheat Lines & Oct. 25 & Nov. 5 & Nov. 15 & Nov. 25 & Dec. 5 & Dec.15 & Mean \\
\hline PR-103 & $18407 \mathrm{~b}-\mathrm{f}$ & $19591 \mathrm{a}-\mathrm{c}$ & $17145 \mathrm{c}-\mathrm{g}$ & $16443 \mathrm{~d}-\mathrm{i}$ & $14332 \mathrm{ij}$ & $9629 \mathrm{~lm}$ & $15428 \mathrm{bc}$ \\
\hline PR-105 & $18369 \mathrm{c}-\mathrm{f}$ & $17628 \mathrm{c}-\mathrm{g}$ & $16332 \mathrm{f}-\mathrm{i}$ & $14887 \mathrm{~h}-\mathrm{j}$ & $14369 \mathrm{ij}$ & $9628 \mathrm{~m}$ & $14569 \mathrm{c}$ \\
\hline PR-106 & $19665 \mathrm{a}-\mathrm{c}$ & $19628 \mathrm{a}-\mathrm{c}$ & $19110 \mathrm{a}-\mathrm{e}$ & $16888 \mathrm{~d}-\mathrm{i}$ & $15369 \mathrm{f}-\mathrm{i}$ & $12258 \mathrm{jk}$ & $16651 \mathrm{a}$ \\
\hline PR-107 & $20776 \mathrm{a}$ & $19924 \mathrm{a}-\mathrm{c}$ & $17961 \mathrm{c}-\mathrm{f}$ & $16406 \mathrm{e}-\mathrm{i}$ & $15036 \mathrm{ij}$ & $10369 \mathrm{~km}$ & $15939 \mathrm{ab}$ \\
\hline PR-108 & $19554 \mathrm{a}-\mathrm{d}$ & $17702 \mathrm{c}-\mathrm{g}$ & $16554 \mathrm{e}-\mathrm{i}$ & $15406 \mathrm{~g}-\mathrm{j}$ & $15258 \mathrm{~h}-\mathrm{j}$ & $9629 \mathrm{~m}$ & $14910 \mathrm{c}$ \\
\hline PR-109 & $20332 \mathrm{ab}$ & $16813 \mathrm{e}-\mathrm{i}$ & $16962 \mathrm{c}-\mathrm{h}$ & $16369 \mathrm{~d}-\mathrm{i}$ & $12925 \mathrm{jl}$ & $7406 \mathrm{~m}$ & $14095 \mathrm{c}$ \\
\hline Mean & $19517 \mathrm{a}$ & $18548 \mathrm{ab}$ & $17344 \mathrm{~b}$ & $16067 \mathrm{c}$ & $14548 \mathrm{~d}$ & $9820 \mathrm{e}$ & \\
\hline
\end{tabular}

Means in the same category following by different letters are significantly different from each other at $5 \%$ level of probability. LSD (sowing dates): 1.08 LSD (wheat advance lines): 0.93 LSD (sowing dates $x$ wheat advance lines): 2.29 .

\section{0-Kernel Weight (G)}

Table 12: Mean values for 1000-kernel weight $(\mathrm{g})$ of newly developed wheat advance lines planted on different sowing dates evaluated at CCRI Pirsabak during 2013-2014.

\begin{tabular}{|c|c|c|c|c|c|c|c|}
\hline \multicolumn{7}{|c|}{ Sowing Dates } \\
\hline Wheat Lines & Oct. 25 & Nov. 5 & Nov. 15 & Nov. 25 & Dec. 5 & Dec.15 & Mean \\
\hline PR-103 & $53.4 \mathrm{a}-\mathrm{c}$ & $49.2 \mathrm{~b}-\mathrm{f}$ & $48.3 \mathrm{~b}-\mathrm{g}$ & $44.0 \mathrm{c}-\mathrm{g}$ & $43.3 \mathrm{c}-\mathrm{g}$ & $39.5 \mathrm{fg}$ & $46.3 \mathrm{~cd}$ \\
\hline PR-105 & $49.1 \mathrm{~b}-\mathrm{f}$ & $47.0 \mathrm{~b}-\mathrm{g}$ & $43.2 \mathrm{c}-\mathrm{g}$ & $42.1 \mathrm{~d}-\mathrm{g}$ & $41.5 \mathrm{~d}-\mathrm{g}$ & $37.9 \mathrm{~g}$ & $43.5 \mathrm{~d}$ \\
\hline PR-106 & $51.1 \mathrm{a}-\mathrm{e}$ & $47.1 \mathrm{~b}-\mathrm{g}$ & $47.2 \mathrm{~b}-\mathrm{g}$ & $46.4 \mathrm{~b}-\mathrm{g}$ & $44.6 \mathrm{c}-\mathrm{g}$ & $39.8 \mathrm{fg}$ & $46.0 \mathrm{~cd}$ \\
\hline PR-107 & $53.0 \mathrm{a}-\mathrm{c}$ & $53.3 \mathrm{a}-\mathrm{c}$ & $50.7 \mathrm{a}-\mathrm{e}$ & $48.5 \mathrm{~b}-\mathrm{g}$ & $48.0 \mathrm{~b}-\mathrm{g}$ & $43.7 \mathrm{c}-\mathrm{g}$ & $49.5 \mathrm{ab}$ \\
\hline PR-108 & $60.7 \mathrm{a}$ & $56.6 \mathrm{ab}$ & $51.6 \mathrm{a}-\mathrm{d}$ & $49.7 \mathrm{~b}-\mathrm{f}$ & $42.1 \mathrm{~d}-\mathrm{g}$ & $41.7 \mathrm{~d}-\mathrm{g}$ & $50.4 \mathrm{a}$ \\
\hline PR-109 & $54.0 \mathrm{a}-\mathrm{c}$ & $49.1 \mathrm{~b}-\mathrm{f}$ & $49.1 \mathrm{~b}-\mathrm{f}$ & $44.6 \mathrm{c}-\mathrm{g}$ & $47.4 \mathrm{~b}-\mathrm{g}$ & $40.6 \mathrm{e}-\mathrm{g}$ & $47.5 \mathrm{bc}$ \\
\hline Mean & $53.5 \mathrm{a}$ & $50.4 \mathrm{ab}$ & $48.3 \mathrm{bc}$ & $45.9 \mathrm{c}$ & $44.5 \mathrm{~cd}$ & $40.5 \mathrm{~d}$ & \\
\hline
\end{tabular}

Means in the same category following by different letters are significantly different from each other at $5 \%$ level of probability. LSD (sowing dates): 2.74 LSD (wheat advance lines): 1.97 LSD (sowing dates $x$ wheat advance lines): 4.83 .

Mean results (Table 12) revealed that 1000-kernel weight was significantly decreased as planting delay. 1000-kernel weight ranged from 49.1g (PR-105) to 60.7g (PR-108), for
Oct. $25^{\text {th }}$ sown crop. As planting delayed, 1000-kernel weight gradually decreased for all wheat advance lines and kernel weight loss continued till Dec. $15^{\text {th }}$. Differences between wheat 
advance lines became prominent with passage of sowing time. The 1000-kernel of wheat lines PR-103, PR-105, PR-106, PR-107, PR-108 and PR-109 were decreased from range of 22.2 to $31.3 \%$ respectively when planting was delayed from Oct. $25^{\text {th }}$ to Dec. $15^{\text {th }}$. Maximum 1000-kernel weight $(50.4 \mathrm{~g})$ was recorded for PR108 and minimum 1000-kernel weight (43.5g) was recorded for PR-105 at all sowing date. 1000-kernel weight is not related with grains per spike in different genotypes. For example, maximum 1000-kernel weight for PR-108 but minimum grains per spike. It shows that genotype strongly affects the grain size. These findings are in line with those of [25] who observed the effect of different sowing times on 1000-kernel weight of wheat. The reasons may be genetic make-up of the genotypes or less/more competition for plant nutrients which produced weak/healthy plants and turn into healthier and plump seed formation. The early sowing resulted in better development of the grains due to longer growing period. These findings are strongly supported by those of Spink et al. [26] and Shahzad et al. [21] who had also reported decreased 1000 -kernel weight with delay in sowing. Dokuyucu et al. [2] reported significant decreases in grain weight on the earlier and later sowing dates. Differences in 1000-kernel weight among varieties might be attributed to their genetic diversity. These results are in line with those of Shahzad et al. [21].

\section{Harvest Index (\%)}

Table 13: Mean values for harvest index (\%) of newly developed wheat advance lines planted on different sowing dates evaluated at CCRI Pirsabak during 2013-2014.

\begin{tabular}{|c|c|c|c|c|c|c|c|}
\hline \multicolumn{9}{|c|}{ Sowing Dates } \\
\hline Wheat Lines & Oct. 25 & Nov. 5 & Nov. 15 & Nov. 25 & Dec. 5 & Dec.15 & Mean \\
\hline PR103 & $28.3 \mathrm{~d}-\mathrm{g}$ & $27.2 \mathrm{~d}-\mathrm{g}$ & $26.9 \mathrm{~d}-\mathrm{g}$ & $28.9 \mathrm{c}-\mathrm{g}$ & $30.4 \mathrm{a}-\mathrm{f}$ & $33.9 \mathrm{a}$ & $29.3 \mathrm{a}$ \\
\hline PR105 & $30.0 \mathrm{~b}-\mathrm{f}$ & $26.6 \mathrm{~d}-\mathrm{g}$ & $26.3 \mathrm{~d}-\mathrm{g}$ & $31.7 \mathrm{a}-\mathrm{e}$ & $32.6 \mathrm{a}-\mathrm{d}$ & $33.3 \mathrm{a}-\mathrm{c}$ & $30.1 \mathrm{a}$ \\
\hline PR106 & $24.7 \mathrm{fg}$ & $29.1 \mathrm{c}-\mathrm{g}$ & $28.6 \mathrm{~d}-\mathrm{g}$ & $31.2 \mathrm{a}-\mathrm{f}$ & $26.3 \mathrm{~d}-\mathrm{g}$ & $32.8 \mathrm{~b}-\mathrm{f}$ & $28.8 \mathrm{a}$ \\
\hline PR107 & $26.3 \mathrm{~d}-\mathrm{g}$ & $28.3 \mathrm{~d}-\mathrm{g}$ & $25.9 \mathrm{~d}-\mathrm{g}$ & $29.0 \mathrm{c}-\mathrm{g}$ & $31.6 \mathrm{a}-\mathrm{e}$ & $33.8 \mathrm{ab}$ & $29.2 \mathrm{a}$ \\
\hline PR108 & $26.5 \mathrm{~d}-\mathrm{g}$ & $27.8 \mathrm{~d}-\mathrm{g}$ & $30.0 \mathrm{~b}-\mathrm{f}$ & $29.1 \mathrm{c}-\mathrm{g}$ & $25.9 \mathrm{~d}-\mathrm{g}$ & $32,7 \mathrm{a}-\mathrm{d}$ & $28.6 \mathrm{ab}$ \\
\hline PR109 & $25.1 \mathrm{e}-\mathrm{g}$ & $22.7 \mathrm{~g}$ & $25.5 \mathrm{e}-\mathrm{g}$ & $22.6 \mathrm{~g}$ & $26.4 \mathrm{~d}-\mathrm{g}$ & $32.7 \mathrm{a}-\mathrm{d}$ & $25.8 \mathrm{~b}$ \\
\hline Mean & $26.8 \mathrm{~b}$ & $26.9 \mathrm{~b}$ & $27.2 \mathrm{~b}$ & $28.8 \mathrm{~b}$ & $28.9 \mathrm{~b}$ & $33.2 \mathrm{a}$ & \\
\hline
\end{tabular}

Means in the same category following by different letters are significantly different from each other at $5 \%$ level of probability. LSD (sowing dates): 3.12 LSD (wheat advance lines): 2.72 LSD (sowing dates $x$ wheat advance lines): 6.67.

Mean results (Table 13) showed that significant differences for harvest index (\%) of various wheat advance lines sown at different dates. Maximum harvest index (33.2\%) was recorded for Dec. $15^{\text {th }}$ sown crop across all sowing dates while the minimum harvest index $(26.8 \%)$ was recorded for Oct. $25^{\text {th }}$ sown crop. There were significant differences among the wheat advance lines for harvest index (\%). Minimum harvest index (25.8\%) was recorded for wheat line PR-109 and maximum harvest index $(30.1 \%)$ was recorded for PR-105 at all sowing dates. The interaction of wheat lines and sowing date for harvest Index (\%) was not significant. Harvest index was increased (19.2\%) for Oct. $25^{\text {th }}$ sown crop as compare to Dec $25^{\text {th }}$. The contrasting results reported by Wajjid [24], who observed that both early and delay planting affect harvest index significantly.

\section{Grain Yield $\left(\mathrm{Kg} \mathrm{Ha}^{-1}\right)$}

Table 14: Mean values for grain yield $\left(\mathrm{kg} \mathrm{ha}^{-1}\right)$ of newly developed wheat advance lines planted on different sowing dates evaluated at CCRI Pirsabak during 2013-2014.

\begin{tabular}{|c|c|c|c|c|c|c|c|}
\hline \multicolumn{9}{|c|}{ Sowing Dates } \\
\hline Wheat Lines & Oct. 25 & Nov. 5 & Nov. 15 & Nov. 25 & Dec. 5 & Dec.15 & Mean \\
\hline PR103 & $5231 \mathrm{a}-\mathrm{f}$ & $5344 \mathrm{a}-\mathrm{e}$ & $4609 \mathrm{~d}-\mathrm{k}$ & $4737 \mathrm{a}-\mathrm{h}$ & $4361 \mathrm{f}-\mathrm{m}$ & $3544 \mathrm{~m}-\mathrm{q}$ & $4637 \mathrm{a}$ \\
\hline PR105 & $5496 \mathrm{a}-\mathrm{c}$ & $4685 \mathrm{c}-\mathrm{j}$ & $4279 \mathrm{~h}-\mathrm{n}$ & $4699 \mathrm{a}-\mathrm{h}$ & $4755 \mathrm{~b}-\mathrm{i}$ & $3432 \mathrm{n}-\mathrm{q}$ & $4599 \mathrm{ab}$ \\
\hline PR106 & $4748 \mathrm{~b}-\mathrm{j}$ & $5662 \mathrm{a}$ & $5474 \mathrm{a}-\mathrm{c}$ & $5273 \mathrm{a}-\mathrm{d}$ & $4013 \mathrm{i}-\mathrm{o}$ & $3633 \mathrm{l}-\mathrm{q}$ & $4800 \mathrm{a}$ \\
\hline PR107 & $5466 \mathrm{a}-\mathrm{d}$ & $5608 \mathrm{ab}$ & $4605 \mathrm{~d}-\mathrm{k}$ & $4727 \mathrm{e}-\mathrm{l}$ & $4744 \mathrm{~b}-\mathrm{j}$ & $3668 \mathrm{l}-\mathrm{q}$ & $4803 \mathrm{a}$ \\
\hline PR108 & $5174 \mathrm{a}-\mathrm{g}$ & $4927 \mathrm{a}-\mathrm{h}$ & $4783 \mathrm{a}-\mathrm{i}$ & $4187 \mathrm{j}-\mathrm{p}$ & $3922 \mathrm{i}-\mathrm{p}$ & $3031 \mathrm{pq}$ & $4341 \mathrm{~b}$ \\
\hline PR109 & $5059 \mathrm{a}-\mathrm{h}$ & $3823 \mathrm{k}-\mathrm{p}$ & $4327 \mathrm{~g}-\mathrm{m}$ & $3673 \mathrm{l}-\mathrm{q}$ & $3327 \mathrm{o}-\mathrm{q}$ & $2870 \mathrm{q}$ & $3847 \mathrm{c}$ \\
\hline Mean & $5196 \mathrm{a}$ & $5008 \mathrm{ab}$ & $4679 \mathrm{~b}$ & $4549 \mathrm{bc}$ & $4187 \mathrm{c}$ & $3366 \mathrm{~d}$ & \\
\hline
\end{tabular}

Means in the same category following by different letters are significantly different from each other at $5 \%$ level of probability. LSD (sowing dates): 4.33 LSD (wheat advance lines): 3.44 LSD (sowing dates $x$ wheat advance lines): 8.44 . 
Mean results (Table 14) showed grain yield $\left(\mathrm{kg} \mathrm{ha}^{-1}\right)$ was significantly decreased as planting delayed. Maximum grain yield (5196kg ha $\left.{ }^{-1}\right)$ was recorded for Oct. $25^{\text {th }}$ sown crop which decreased significantly to (3366 $\mathrm{kg} \mathrm{ha}^{-1}$ ) for Dec. $15^{\text {th }}$ sown crop. Among time of sowing, significant maximum grain yield was recorded (5196 $\left.\mathrm{kg} \mathrm{ha}^{-1}\right)$ and $\left(5008 \mathrm{~kg} \mathrm{ha}^{-1}\right)$ for Oct. $25^{\text {th }}$ and Nov. $05^{\text {th }}$ against minimum grain yield $\left(3366 \mathrm{~kg} \mathrm{ha}^{-1}\right)$ for Dec. $15^{\text {th }}$ sown crop. Thus, wheat advance lines should be preferably sown on Oct $25^{\text {th }}$ and Nov. $05^{\text {th }}$ as optimum sowing time for maximum grain yield in KP zone. Average performance of wheat lines PR-107 and PR-103 were recorded (5537 $\left.\mathrm{kg} \mathrm{ha}^{-1}\right)$ and (5287kg ha $^{-1}$ ) followed by PR-106 (5208kg ha-1), while PR-109 produce lowest grain yield ( $\left.4441 \mathrm{~kg} \mathrm{ha}^{-1}\right)$, when wheat lines were sown on Oct. $25^{\text {th }}$ and Nov. $5^{\text {th }}$. Averaged performance of wheat lines PR-107 and PR-106 produce highest grain yield (4803kg ha-1), (4800 kg ha-1) followed by PR-103.while PR-109 produce the lowest grain yield ( $\left.3847 \mathrm{~kg} \mathrm{ha}^{-1}\right)$ at all sowing dates. This may be attributed to the smaller number of grains spike ${ }^{-1}$, spikelets spike $^{-1}$, lower biomass yield and smaller grain size. Therefore, wheat lines PR-107 and PR-106 produce higher grain yield at all planting dates as these lines has been released in future for normal and late sowing in irrigated areas of KP zone. Grain yield decreased significantly in all wheat advance lines with delay in sowing time. The percent decreased in wheat lines from Oct. $25^{\text {th }}$ to delayed till Dec. $15^{\text {th }}$ sown crop were $32.2 \%$ in PR-103, $37.5 \%$ in PR-105, 23.4\% in PR 106, 32.8\% in PR-107, 41.4\% in PR-108 and 43.2\% respectively in PR-109. Grain yield losses due to late planting were recorded $3.6 \%$ when planting was done on Nov. $5^{\text {th }}, 9.9 \%$ when planting was done on Nov. $15^{\text {th }}, 12.4 \%$ when planting was done on Nov. $25^{\text {th }}$ and $19.4 \%$ when planting was done on Dec. $5^{\text {th }}$ and $35.2 \%$ respectively when planting was further delayed till Dec. $15^{\text {th }}$. These results are in line with those [27-30] who reported that sowing times and varieties of wheat both significantly effect on grain yield. Decrease in the yield attributes i.e. spike length spikelets spike ${ }^{-1}$ number grains spike ${ }^{-1}$ and 1000-kernel weight due to delay in sowing contributed to decrease in the grain yield for which the main reasons are the decrease in growing degree days [31-35], longer photoperiod and higher temperature during the reproductive stage and the genotype. The yield attributes studied in this experiment showed smaller decrease $11.3 \%$ in number of grains spike ${ }^{-1}$ and $24.2 \%$ in 1000 -kernel weight as compared with the $35.2 \%$ decreased in the grain yield when sowing was delayed from Oct. $25^{\text {th }}$ to Dec. $15^{\text {th }}$. It shows that the rest of the decreased in grain yield might be due to decrease in the number of productive tillers per unit area. In later sowing date's biomass and grain yield decreased due to undesired environmental conditions and lack of suitable transforming preserved matters to seeds as a result of increasing temperature at the end of growth season [36-38].

\section{Conclusion}

It is concluded from the results of the experiment that sowing in time around from Oct. $25^{\text {th }}$ to Nov. $05^{\text {th }}$ in central KP is suitable for policy for getting higher grain yields and even the wheat advance lines cannot be recommended for late sowing due to yield losses in delay sowing. Significant differences exist among wheat advance lines in maturity duration. So breeders should select those genotypes, which could compensate up to large extent, although it may not recover fully, yield losses due to delay in sowing. Among different sowing dates, significant maximum grain yield was recorded (5196kg ha-1) and (5008 $\left.\mathrm{kg} \mathrm{ha}^{-1}\right)$ for Oct $25^{\text {th }}$ and Nov. $05^{\text {th }}$ against the minimum grain yield of $(3366 \mathrm{~kg} \mathrm{ha}$ $\left.{ }^{1}\right)$ for Dec. $5^{\text {th }}$ sown crop, we recommend that all wheat advance lines may be preferably sown on Oct $25^{\text {th }}$ to Nov. $05^{\text {th }}$, indicating optimum sowing time for producing maximum grain yield in KP zone [39]. Wheat advance lines PR-107 and PR-103 sown on Oct. $25^{\text {th }}$ and Nov. $5^{\text {th }}$ resulted maximum grain yield recorded $\left(5537 \mathrm{~kg} \mathrm{ha}^{-1}\right)$ and $\left(5287 \mathrm{~kg} \mathrm{ha}^{-1}\right)$ respectively as compare to the rest of advance lines, while PR-109 produce minimum grain yield ( $4441 \mathrm{~kg} \mathrm{ha}^{-1}$ ) sown on Oct. $25^{\text {th }}$ and Nov. $5^{\text {th }}$. So we recommend that wheat advance lines PR-107 and PR-103 may be preferably sown on Oct $25^{\text {th }}$ to Nov. $05^{\text {th }}$, indicating optimum sowing time for producing maximum grain yield in KP zone. Among wheat advance lines on over all planting dates, PR-107, PR-106, PR-103 and PR-105 produced higher average yield especially PR-107 and PR-106 recorded (4803 $\left.\mathrm{kg} \mathrm{ha}^{-1}\right)$ and (4800 $\left.\mathrm{kg} \mathrm{ha}^{-1}\right)$ maximum yield. PR-103 and PR-105 were recorded (4637 kg ha-1) and (4599kg ha-1) respectively, while PR-109 recorded minimum yield (3847kg ha-1).

\section{Recommendation}

Therefore, wheat advance lines PR-107, PR-106 and PR103 are recommended in future for normal and late sowing in irrigated areas of KP zone for maximum grain yield. These all lines will be released as new varieties after further testing in regional and national trials.

\section{References}

1. Murungu FS, Madanzi T (2010) Seed priming, genotype and sowing date effect on emergency, growth and yield of wheat in a tropical low altitude area of Zimbanwe. African Journal of Agriculture Research 5(17): 2341-2349.

2. Dokuyucu T, Akkaya A, Yigitoglu D (2004) The effect of different sowing dates on growing periods, yield and yield components of some bread wheat (Triticum aestivum L) cultivars grown in the East- Mediterranean Region of Turkey. Journal of Agronomy 3(2): 126-130.

3. Tanveer SK, Hussain L, Asif M, Mujahid MY, Muhammad S, et al. (2009) Performance of different wheat line as affected by different planting dates and seeding rates under high rainfall after of potohar. Pakistan Journal of Agriculture Science 46(2): 102-106.

4. Dabre WM, Lall SB, Lngole GL (1993) Effects of sowing dates on yield, ear number, stomatal frequency and stomatal index in wheat. J Maharashatra Agri Univ 8: 64-66.

5. Qamar M, Shafiullah, Makeen S (2004) Genetic variability among wheat cultivars and effect of planting date on grain and straw yield under double cropping zone of Northern areas of Pakistan. Sarhad J of Agri 20(1): 99-102.

6. Singh T, Dhaliwal GS (2000) Performance of wheat varieties under late sowing conditions in south western region of Punjab. J of Res Pb Agri Univ 37(3-4): 181-183. 
7. Subhan F, Khan M, Jamro GH (2004) Effect of different planting date, seeding rate and weed control method on grain yield and yields components in wheat. S J of Agri 20 (1): 51-55.

8. Kumar R, Sharma SN (2003) Effect of levels of nitrogen on wheat as influenced by date of sowing. Annals of Agri Res 24(1): 104.

9. Singh S, Pal M (2003) Growth, yield and phonological response of wheat cultivars to delayed sowing. Ind J of PP Phys 8(3): 277-286.

10. Patil KS, Durge DV, Phadnawis BN, Shivankar RS, Rathod TH (2000) Effect of sowing dates on biomass production of wheat cultivars. Ann of Pl Phys 14(2): 115-119.

11. Ahmed M, Arain MA, Siddiqui KA (1994) Effect of contemporary rotation simulation on the grain weight, protein and lysine content of bread wheat (Triticum aestivum L). Pak J of Bot 26(2): 311-339.

12. Subhan F, Khan M, Jamro GH (2003) Weed management through planting date, seeding rate and weed control method in wheat. Pakistan Journal of Weed Sciences Research 9(1-2): 49-57.

13. Qasim M, Qamer M, Faridullah M, Alam M (2008) Sowing dates effect on yield and yield components of different wheat cultivars. Journal of Agriculture Research 46(2): 135-140.

14. Khan MA, Anwer J, Sattar A, Akhter MA (2001) Effect of seed rate on wheat yield under different sowing dates and row spacing. J Agric Res 39: $223-253$.

15. Subhan F, Ahmaed N, Anwar M, Shah NH, Siddiq M, et al. (2004) Response of newly developed wheat cultivars/advance lines to planting dates in the central Agro-ecological zones of NWFP. Asian J Plant Sci 3(1): 87-90.

16. Sial MA, Arain MA, Javed, Nizamani NA (2001) Response of wheat genotypes on yield and yield components with changing plant population densities. Pak J Bot 33: 797-800.

17. Slafer GA, Whitechurch EM (2001) Manipulating wheat development to improve adaptation. In Application of Physiology in Wheat Breeding. Reynolds, North America.

18. Slafer GA, Rawson HM (1994) Sensitivity of wheat phasic development to major environmental factors: A re-examination of some assumptions made by physiologists and modellers. Aust J Plant Physiol 21(4): 393 426

19. Shafiq HM (2004) Modeling growth, radiation use efficiency and yield of wheat at different sowing dates and nitrogen levels under arid conditions of Bhawalpur. University of Agriculture, Faisalabad, Pakistan.

20. Hussain A (1995) Wheat and barley varieties of Pakistan. National Seed Registration Dept Ministry of Food, Agric and Livestock, Pakistan.

21. Shahzad K, Bakht J, Shah WA, Shafi M, Jabeen N (2002) Yield and yield components of various wheat cultivars as affected by different sowing dates. Asian J PI Sci 1(5): 522-525.

22. Haider RS (2004) Growth and yield response of three wheat varieties to $\mathrm{N}$ alone and in combination with $\mathrm{P}$ and $\mathrm{P}+\mathrm{K}$ under late sown conditions. Agri Thesis, University of Agricuture, Faisalabad, Pakistan.

23. Ahmad IZ (1991) Effect of irrigation and cultivars on the growth and yield of wheat. Agri Thesis, University of Agriculture, Pakistan.
24. Wajid SA (2004) Modeling development, growth and yield of wheat under different sowing dates, plant populations and irrigation levels. Department of Agron Univ of Arid Agri, Pakistan.

25. French RJ, Schultz JE, Rudd CL (2005) Effect of time of sown on wheat phenology in South Australia. Austral J Exp Agric Anim Husb 19(96): 89-96.

26. Spink JH, Semere T, Sparkes DL, Whaley JM, Foulkers MJ, et al. (2000) Effect of sowing date on plant density of winter wheat. Ann App Biol 137(2): 179-188.

27. Sandhu IS, Sharma AR, Sur HS (1999) Yield performance and heat unit requirement of wheat varieties as affected by sowing dates under rainfed conditions. Indian J Agric Sci 69: 175-179.

28. Tahir M, Ali A, Nadeem MA, Hussain A, Khalid F (2009) Effect of Different Sowing Dates on Growth and Yield of Wheat Varieties in District Jhang Pakistan. Pak J life soc sci 7(1): 66-69.

29. Abdullah M, Rehman A, Ahmad N, Rasul I (2007) Planting time effect on Grain and quality characteristics of wheat. Pak J Agri Sci 44(2): 211231.

30. Akhtar M, Ahmad N, Nasrullah M, Ali B, Zahid AR, et al. (2012) Effect of late planting on emergence, tillering and yield of the various varieties of wheat. J of Ani \& Pl Sci 22(4): 1163-1166.

31. Anwar J, Khan SB, Rasuli I, Zulkiffal M, Hussain M (2007) Effect of sowing dates on yield and yield components in wheat using stability analysis Int. J of Agri \& Bio 1560-8530.

32. Akdamar M, Tayyar S, Gokkus A (2002) Effects of different sowing times on yield and yield related traits in bread wheat grown in Canakkale. Akdeniz universitesi ziraat fakultasi Dergisi 15(2): 81-87.

33. Anjum M, Ali M, Ali Q, Mohy U (2004) Determination of grain yield of different wheat varieties as Influenced by planting dates in AgroEcological Conditions of Vehari. Pak J life soc Sci 2(1): 5-8.

34. Aslani F, Mehrvar MR (2012) Responses of wheat genotypes as affected by different sowing dates. Asian journal of Agri Sci 4(1): 72-74.

35. Hanson B (2001) Planting drate influence on yield and agronomic traits of hard red spring wheat in north eastern North Dakota. Langdon Res Ext Center NDSU Agric Report I.

36. Inamullah NH, Shah Z, Haq F, Ullah K (2007) An analysis of the planting date effect on the yield and yield attributes of spring Sarhad. J Agri 23(2): 270-275.

37. Khosravi V, Nejad GK, Nejad GM, Yousefi K (2010) The effect of different sowing dates on yield and yield components of wheat Cultivars. Inter J of Agro and Prod 1(3): 77-82.

38. Lak M, Farnia A, Shaban M (2013) Effect of different sowing dates on yield components of wheat cultivars in Lorestan provience, Iran. Adv Agri Biol 1(4): 89-93.

39. Sial MA, Arain MA, Dahoti MU, Markhand GS, Laghari KA, et al. (2010) Effect on sowing dates on yield components on mutant-cum-hybrid lines of bread wheat. Pak J Bot 42(1): 269-277 
This work is licensed under Creative Commons Attribution 4.0 License DOI: 10.19080/ARTOAJ.2017.07.555705
Your next submission with Juniper Publishers will reach you the below assets

- Quality Editorial service

- Swift Peer Review

- Reprints availability

- E-prints Service

- Manuscript Podcast for convenient understanding

- Global attainment for your research

- Manuscript accessibility in different formats ( Pdf, E-pub, Full Text, Audio)

- Unceasing customer service

Track the below URL for one-step submission https://juniperpublishers.com/online-submission.php 